\title{
Ein Beitrag zur Kenntniss der spinalen Lähmung bei Neugeborenen.
}

Von

\author{
C. C. Th. Litzmann.
}

Ich knüpfe meine Bemerkungen über diesen Gegenstand an die Mittheilung eines kürzlich von mir beobachteten Falles.

Frau N. N. aus Hamburg wurde im October 1878 von ihrem Hausarzte mit der Frage an mich gewiesen, ob ich in ihrer gegenwärtigen Schwangerschaft die Einleitung einer Frühgeburt für rathsam halte. Sie hatte ausser einem Abortus bereits neun rechtzeitige Geburten überstanden. Die ersten fünf Kinder waren in Schädellage lebend zur Welt gekommen; von diesen fünf Geburten waren zwei natürlich verlaufen, drei mit der Zange beendigt. Bei der Geburt des sechsten Kindes war die Nabelschnur neben dem Kopfe vorgefallen; das mit der Zange extrahirte Kind war todt. Bei den folgenden drei Geburten musste die Extraction an den Füssen gemacht werden, ein Mal bei ursprünglicher Fusslage, zwei Male nachdem wegen Querlage das Kind auf die Füsse gewendet war. Die Extraction war schwierig, namentlich die Entwickelung des Kopfes, und die Kinder, angeblich grösser als die früheren, starben während der Operation. Sämmtlichen Geburten gemeinsam war ein beträchtlicher Wassergehalt des Uterus und Wehenschwäche; nur bei der siebenten, einer Fussgeburt, waren die Wehen kräftig, so dass die Austreibung des Kindes bis zum Kopfe rasch von Statten ging. Bei dieser, sowie bei der letzten Geburt, war das Fruchtwasser vorzeitig abgegangen, bei allen übrigen die Fruchtblase durch den Arzt gesprengt. Die Placenta hatte, mit Ausnahme der zweiten, natürlich verlaufenen Schädelgeburt und der Fussgeburt, jedesmal künstlich gelöst werden 
müssen. Die Wochenbetten waren im Ganzen normal verlaufen; nach der letzten Geburt war ein im Uterus zurückgebliebenes Placentarstück nachträglich in verjauchtem Zustande ausgestossen.

Die 39jährige kleine, übrigens regelmässig gewachsene Frau war in ihrer Kindheit scrophulös, nicht rachitisch gewesen und hatte frühzeitig gehen gelernt. Die Menstruation war schon im 13. Jahre regelmässig eingetreten. Ihre Mutter hatte sieben lebende Kinder geboren. Sämmtliche Geburten waren schwer und erforderten Kunsthülfe. Nach der ersten, mit der Zange beendigten Geburt blieb eine lähmungsartige Schwäche beider Beine zurück, die sich zwar besserte, aber nie wieder ganz verlor und nach jeder Geburt roribergehend wieder zunahm. Drei Schwestern hatten lebende Kinder ohne Kunsthülfe geboren. Die Untersuchung des Beckens ergab kleine äussere Quermaasse: Spinae ilei $24,9 \mathrm{~cm}$, Cristae ilei $28,5 \mathrm{~cm}$. Die vordere Beckenwand war mässig hoch, nicht sehr geneigt; beide Seitenwände gleichmässig abgeflacht und leicht zu bestreichen; das Promontorium mässig hochstehend, darïber hinaus der letzte, unter einem stumpfen Winkel zurücktretende Lendenwirbel noch eine Strecke weit zu verfolgen. Die Conjugata diagonalis maass ich zu $10,5 \mathrm{~cm}$. Ich. hielt das Becken für ein gleichmässig allgemein verengtes und schätzte die Conjugata vera auf etwa $9 \mathrm{~cm}$.

Die letzte Menstruation war am 9. Mai erschienen; Kindesbewegungen hatte die Schwangere zuerst am 11. September gefühlt, mithin war der rechtzeitige Eintritt der Geburt am 15. Februar 1879 zu erwarten. Ich schob die Entscheidung uiber die Einleitung einer Frühgeburt noch bis zu Anfang Januars 1879 hinaus, wo sich hoffen liess, über das Lage- und Grössenverhältniss der Frucht zum Becken mit grösserer Sicherheit urtheilen zu können. Bei der am 9. Januar wiederholten Untersuchung fand ich die anscheinend mittelgrosse Frucht in dem sehr dünnund schlaffwandigen, nur mässig wasserhaltigen Uterus oberhalb des kleinen Beckens sehr schräg gelagert, die Bauchfläche nach vorn und oben gekehrt, den Kopf auf die rechte Darmbeinschaufel abgewichen. Die Vaginalportion mit eben fuir den Finger durchgängigem Muttermunde stand sehr hoch, ein Kindestheil war von. der Scheide her nicht zu erreichen. Es war demnach abermals eine Complication mit fehlerhafter Kindeslage und Wehenschwäche zu fürchten. Unter diesen Umständen glaubte ich, dass die allerdings bedeutenderen mechanischen Schwierigkeiten bei einer recht- 
zeitigen Geburt gegenüber den Vortheilen, die eine Frühgeburt in dieser Beziehung darbot, durch die entwickeltere Contractionskraft des Uterus und die grössere Widerstandsfähigkeit des Kindes am Ende der Schwangerschaft völlig aufgewogen würden und rieth deshalb von einer Unterbrechung derselben ab.

Da gewünscht wurde, dass ich die Geburt leite, so siedelte die Schwangere am 8. Februar nach Kiel über. Die Wandungen des Uterus erschienen mir jetzt etwas dicker und gespannter; die Frucht lag nicht mehr ganz so schräg, mit der Rückenfläche nach links gekehrt, der Kopf über der rechten Beckenhälfte nach vorn vorspringend. Das untere Uterinsegment hatte sich tiefer ins Becken herabgesenkt, der noch lange Cervicalkanal war für den Finger bequem durchgängig, über dem inneren Muttermunde fühlte ich keinen Kindestheil, doch konnte ich vom vorderen Scheidengewölbe und den Bauchdecken her den Kopf zwischen beide Hände fassen. Ordination: Hängeleibbinde.

Schon am Morgen des 10. Februar stellten sich leise Wehen ein, die gegen Abend an Stärke zunahmen. Um 10 Uhr Nachts kamen sie zwar noch selten, waren aber ziemlich kräftig. Die Fruchtlage war eine andere geworden. Der Kopf lag links im Grunde, der Rumpf in zweiter Diagonale mit der Rückenfläche nach links, rechts über dem Becken der Steiss. Während der Wehe wölbte sich die Fruchtblase ziemlich tief in den schon merklich erweiterten Cervicalkanal vor. Einen Kindestheil konnte ich von der Scheide her nicht erreichen.

Die Wehen wurden zeitweise häufiger und stärker, während der Befund bei der äusseren Untersuchung unverändert blieb. Um 3 Uhr Nachts (11. Februar) war der Cervicalkanal zu einem Cylinder von etwa $8 \mathrm{~cm}$ im Durchmesser erweitert, die gespannte Fruchtblase ragte bis in das Niveau des äusseren Muttermundes herab, in derselben fühlte ich beide Füsse, die Fersen nach links und vorn gerichtet. Um wo möglich noch vor dem Blasensprunge den Eintritt des Steisses ins Becken zu bewirken, liess ich die Kreissende sich auf die rechte Seite legen.

Die Wehen hielten in gleicher Weise an, starke und schmerzhafte mit schwächeren wechselnd. Um 4 Uhr Nachts drängte sich die Spitze der Fruchtblase fast bis in die Schamspalte vor. Die Seitenlage hatte nicht den gewünschten Frfolg gehabt; im Gegentheile hatten sich die Füsse mehr zurückgezogen, so dass ich nach rechts in der Höhe des Beckeneinganges die Fersen nur eben 
noch erreichen konnte. Ich beschloss daher die Extraction nicht länger hinauszuschieben. Nachdem die Kreissende auf ein Querbett gebracht war, stach ich die Fruchtblase mit einer Stricknadel an und liess das Fruchtwasser langsam abfliessen. Die rechte Seite des Kindes lag vor, der Rücken nach vorn, der rechte Arm über dem inneren Muttermunde, auf der rechten Darmbeinschaufel der Steiss mit angezogenen Füssen, die Fersen nach links und vorn gerichtet; etwas höher und nach hinten glaubte ich die quer durch die Uterushöhle gespannte Nabelschnur zu erkennen. Die Kreissende wurde auf ihren Wunsch, wie es bei allen früheren Entbindungen geschehen war, leicht chloroformirt. Ich streckte den zu unterst gelegenen rechten Fuss bis in die Schamspalte herab; er bewegte sich auf Reizung der Fusssohle sehr lebhaft. Trotz einer kurz vorher gemachten Ergotininjection und fortgesetzter Reibungen des Uteringrundes hörten jetzt die Wehen fast gänzlich auf. Wenige Minuten später rief mein Assistent, Herr Dr. Werth, der neben der Chloroformirung die Ueberwachung des Uterus übernommen hatte, mir zu, dass der Fötalpuls auffällig langsam werde. Zugleich bemerkte ich, dass der herabgestreckte Fuss auf Reize nicht mehr reagirte. Ohne Hoffnung, das Kind zu retten, unternahm ich die Extraction, von den Wehen so gut wie gar nicht unterstützt. Die Einleitung des Steisses ins Becken war mühsam und erforderte einen ziemlichen, jedoch keineswegs übermässigen Kraftaufwand. Dabei hörte ich zwei Male das allen Geburtshelfern bekannte leise Knacken in den Beingelenken, welches so häufig, schon bei mässig starkem Zuge an den Schenkeln, durch die Dehnung der Kapselbänder entsteht. Der linke Fuss trat zugleich mit ein und wurde dann ebenfalls herabgestreckt, Beim Austritt des Steisses ging kein Meconium ab. Die Rückenfläche hatte sich von links her nach vorn und rechts gewandt. Die Lösung der Arme machte keine Schwierigkeit. Ungeachtet des ron meinem Assistenten ron Anfang an auf den Grund der Gebärmutter ausgeïbten Druckes hatte sich der Uteruskörper während der Extraction des Rumpfes gänzlich über die Frucht zurückgezogen, so dass zuletzt der Druck durch die dünne Cerricalwand direct gegen den Kopf gerichtet werden konnte. So fand ich denn zu meiner Freude das Kinn schon unter dem Niveau des Beckeneinganges, eben links rom Promontorium, zog es rasch durch einen senkrechten Zug herab und entwickelte den Kopf durch den gewöhnlichen (L evret'schen) Handgriff ohne besonderen Aufwand von Zeit und Kraft. Die 
sehr dünne Nabelschnur war in mehrfachen Windungen um Brust und Hals geschlungen. Obwohl nach dem Austritte des Steisses der Fötalpuls nicht mehr gehört war, zeigte das Kind - ein völlig reifes Mädchen von mittlerer Grösse - noch schwachen Herzschlag und Nabelschnurpuls, es war auch nicht auffällig bleich, die Glieder nicht schlaff, und schon vor der Abnabelung erfolgte eine spontane, ziemlich tiefe, rasselnde Inspiration. Im warmen Bade wurde es, wenn auch erst nach längerer Zeit, zum regelmässigen Athmen und lauten Schreien gebracht. Am Kopfe waren keine Druckspuren vom Becken sichtbar.

Eine ziemlich starke Blutung aus dem Uterus wurde durch Massage bald gestillt. Doch zeigte derselbe eine grosse Neigung, wieder zu erschlaffen. Erst nach einer einstündigen unausgesetzten Ueberwachung entwickelten sich kräftigere Wehen ohne Zunahme der Blutung. Die Placenta blieb zurück. Die aus der Narcose erwachte Entbundene fühlte sich anfangs ganz frisch und glücklich im Besitze eines lebenden Kindes. Bald aber fing sie, ohne neue Blutung, an zu frösteln, Uebelkeiten und Gähnen stellten sich ein, der Puls wurde sehr langsam (Wein). Ich löste die rechts noch theilweise adhärente Placenta ohne Mühe und entfernte nachträglich noch einen Eihautfetzen und einige Blutcoagula aus dem Uterus. Nach einer Ergotininjection und Ausspülung des Eterushöhle mit heissem Carbolwasser wurde die Entbundene, die sich inzwischen völlig erholt hatte, in ihr gewärmtes Bett zurückgetragen.

Das Wochenbett verlief für die Mutter ohne jegliche Störung. Dagegen gab der Zustand des Kindes sehr bald zu ernster Besorgniss Grund. Schon im Laufe des Vormittags wurde es sehr unruhig, schrie viel, dabei verlor die Stimme merklich an Kraft. Von Zeit zu Zeit zuckte es mit einer eigenthümlich klingenden, krampfhaften Inspiration zusammen, die Arme starr von sich streckend. Am Nachmittage fand ich bei Besichtigung des Körpers beide Beine völlig gelähmt, die Empfindung, wie die Reflexerregbarkeit derselben aufgehoben. Auch die Bauchmuskulatur erschien mir schlaffer, als gewöhnlich, der Bauch aufgebläht. Harn war entleert worden: Die Unruhe und die krampfhaften Erscheinungen verloren sich schon im Laufe der nächsten Tage. Die Darmausleerungen waren von Anfang an mehr gelb gefärbt; sie erfolgten träge, in kleinen Portionen, ohne rechten Drang. Auch die Blase wurde offenbar unvollkommen entleert. Denn 
obwohl das Kind in Zwischenräumen die Windeln nässte, floss bei Druck auf die Unterbauchgegend doch jedesmal Urin aus der Harnröhrenmündung ab. Es wurde der Wärterin anempfohlen, beim Bewegen des Kindes möglichst behutsam zu sein und für die grösste Reinlichkeit zu sorgen. Uebrigens gedieh dasselbe an der Brust einer Amme vortrefflich. Am zehnten Tage bemerkto ich zuerst am linken Fusse auf Reizung der Fusssohle eine langsame und schwache Bewegung der Zehen; einige Tage später auch am rechten. Als die Eltern am 18. März mit dem Kinde nach Hamburg zurückkehrten, hatte der Muskeltonus der Beine entschieden zugenommen, die Reflexbewegungen waren kräftiger geworden und auf die Unterschenkel verbreitet; auch schienen die gelähmten Theile gegen Nadelstiche nicht mehr ganz so unempfindlich, wie früher. Am linken Fusse bestand ein gewisser Grad permanenter Plantarflexion. Die Bauchmuskulatur war immer noch schlaffer, als in der Norm. Doch exfolgten die Darmausleerungen unter sichtlich stärkerem Drange, und der Urin wurde jetzt im Strahle ausgetrieben.

Nach einem Berichte des Hausarztes vom 4. Mai waren die Lähmungserscheinungen in der ersten Zeit nach der Rückkehr unverändert geblieben. Der Versuch, durch Guttaperchaschienen der weiteren Ausbildung eines Spitzfusses vorzubeugen, hatte aufgegeben werden müssen, da der Verband, trotz tüglicher Erneuerung, Druckbrand zu erzeugen drohte. Man hatte sich daher darauf beschränkt, mehrmals am Tage die Haltung des Fusses eine Zeit lang mit der Hand zu rectificiren. Ausserdem wurden spirituöse Waschungen der Beine und Einreibungen ron Unguent. Kalii jodati längs der Wirbelsäule in Anwendung gezogen. Von der Mitte des April ab begann die Reflexerregbarkeit sich deutlich zu heben, scheinbar auch ein wenig die Sensibilität. Das Schreien wurde kräftiger and langathmiger. Als ich am 25. Mai das Kind wieder sah, fand ich es sehr gewachsen; es hatte in der Woche durchschnittlich $125 \mathrm{~g}$ an Gewicht gewonnen. Der Ernährungszustand der Beine entsprach dem des übrigen Körpers, ihr Fleisch war fester geworden. Auch die Bauchmuskulatur erschien mir straffer. Harn und Fäces wurden in normaler Weise entleert. Die Beine bewegten sich auf Reizung in grösserem Umfange, als früher. Strich man z. B. mit der Hand über die Hinterbacken, so zog das Kind die Oberschenkel an den Leib. Bisweilen schien es sogar, als ob es sie willkurlich bewegte. $O b$ und in wie weit das 
Empfindungsvermögen dieser Theile wieder hergestellt sei, darüber konnte ich kein sicheres Urtheil gewinnen. Die Plantarflexion hatte leider an beiden Füssen zugenommen. Es wurde verabredet, die Füsse während der Nacht zu schienen und am Tage wiederholt mit der Hand eine Zeit lang in die normale Haltung zu bringen, daneben mit den Einreibungen des Rückens und den Waschungen der Beine fortzufahren und die gelähmten Glieder sanft zu massiren.

Am 2. Juli wurde zuerst auf den Rath des Herrn Professor Erb, dem ich schon früher eine Mittheilung über den Fall gemacht hatte, eine Untersuchung speciell der elektrischen Erregbarkeit der unteren Extremitäten bei der kleinen Kranken von Herrn Dr. Eisenlohr in Hamburg vorgenommen, deren Ergebniss ich mit seiner gütigen Erlaubniss hier mittheile. Noch immer, schreibt er, besteht eine ziemlich absolute Lähmung der sämmtlichen Muskeln beider Beine. Sie fühlen sich schlaff an, mit Ausnahme des linken Musculus gastrognemius, dessen Contraction sich jedoch leicht überwinden lässt. Besonders schlaff, wohl auch an Volumen etwas reducirt, erscheint die Muskulatur an der Streckseite beider Oberschenkel und an der Aussenseite der Unterschenkel. Reflexe werden von den Fusssohlen ausgelöst in den Muskeln der Beugeseite der Oberschenkel und den Adductoren, am Unterschenkel auch in den Wadenmuskeln; sie fehlen vollkommen in dem Gebiete der Musculi peronei, speciell im Musculus tibialis anticus. Die Sensibilität erscheint ebenfalls herabgesetzt, da ziemlich starkes Kneipen und Stechen keine Reaction hervorrief. Bald machte allerdings ein ununterbrochenes Schreien jedes Urtheil über sensible Perception unmöglich. Die Blase entleerte sich während der Untersuchung kräftig.

Die elektrische Prüfung ergab: die faradische - indirecte und directe - Erregbarkeit ist aufgehoben in beiden CruralisGebieten. Ebenso sind beide Nervi peronei hinter dem Capitulum fibulae unerregbar; von den Muskeln ihres Gebietes reagiren nicht, auch bei directer Reizung, die Musculi tibiales antici beiderseits, rechts auch die übrigen Muskeln nicht, während links bei starkem Strome die Musculi peronei sich contrahiren. Im Ischiadicus-Gebiete sind die Muskeln an der Beugeseite des Oberschenkels vollkommen gut faradisirbar. Ebenso ist im Gebiete der Nervi tibiales die indirecte sowohl, als die directe faradische Erregbarkeit der Wadenmuskeln nicht merklich verringert. Auf- 
fallend war bei der Application der Elektrode am linken Unterschenkel eine länger dauernde Contractur seiner Flexoren, wodurch das sonst schlaffe Knie in leichter Winkelstellung fixirt wurde.

Bei der Untersuchung der galvanischen Erregbarkeit zeigen sich die Streckmuskeln des Unterschenkels von den Nervi crurales aus beiderseits unerregbar, während sie bei directer Reizung, wenn die Anode über der Quadriceps-Sehne stand, eine schwache träge An. S. Z. und Ká S. Z. gaben. Bei Reizung des Nervus peroneus tritt rechts in keinem der zugehörigen Muskeln eine Contraction auf, während von einer gewissen Stromstärke $a b$ durch Stromschleifen, wo auch die Elektrode am Unterschenkel stand, Contractionen der Antagonisten, Plantarflexion des Fusses, der Zehen erfolgten. Die Zuckung in den genannten Muskeln hatte durchaus den normalen, blitzähnlichen Charakter. Eine qualitativ veränderte Zuckung bei directer Reizung konnte im Musculus tibialis anticus, auch bei ziemlich hohen Stromstärken, nicht wahrgenommen werden. Links reagiren auf Reizung des Nervus peroneus noch der Musculus peroneus longus und brevis in qualitativ normaler Weise; dagegen der Musculus tibialis anticus weder auf indirecte, noch auf directe Reizung. Im Ischiadicus-Gebiete zeigen die Beugemuskeln des Unterschenkels, ebenso im Gebiete der Nervi tibiales die Wadenmuskeln beiderseits eine qualitativ und quantitativ normale Reaction.

Zu der nach dieser Untersuchung angerathenen elektrischen Behandlung konnten indess die Eltern des Kindes sich nicht entschliessen, ihre Zustimmung zu geben. So wurde in der bisherigen. Weise fortgefahren. Das Kind nahm dabei erfreulich zu, war geistig rege und heiteren Sinnes. Zu Ende Septembers bekam es den ersten Zahn. Die Beine wurden allmälig etwas belebter, das Kind bewegte sie bäufiger willkürlich und reagirte schon auf gelinde Reizung derselben. Im Ganzen erschien das linke Bein kräftiger, als das rechte.

Eine am 16. October von Herrm Dr. Eisenlohr wiederholte Prüfung der galvanischen Erregbarkeit ergab wesentlich dasselbe Resultat, wie bei der ersten Untersuchung. Die Eltern willigten jetzt in eine elektrische Behandlung des Kindes. Ueber die Art derselben und den bisherigen Erfolg schreibt mir Herr Dr. Eisen lohr unter dem 14. December: wöchentlich drei Sitzungen; 1) Galvanisation des wahrscheinlichen Krankheitsherdes, grosse 
Plattenelektroden, Anode auf die Lumbal- und Sacralgegend, zweite Elektrode auf das Abdomen; 2) R. M., absteigend Lumbalund Dorsalwirbelsäule, ebenfalls grosse Platten; im Ganzen etwa fünf Minuten; 3) labile Erregung der Nerven und Muskeln der unteren Extremitäten mit Kathode (Anode auf das Kreuzbein) und Volta'schen Alternativen. Irgend eine nachtheilige (aufregende) Wirkung wurde nie beobachtet. Das Kind entwickelt sich prächtig. Die letzten Sitzungen mussten allerdings wegen eines mit dem Zahnen verbundenen fieberhaften Zustandes ausgesetzt werden. In dem Verhalten der elektrischen Erregbarkeit lässt sich bis jetzt noch kein wesentlicher Fortschritt erkennen; auch nicht in der Motilität der von vorn herein meist, resp. absolut gelähmten Muskeln. Unläugbar aber ist eine Besserung in der Motilität derjenigen Muskeln, die ihre Erregbarkeit bewahrt hatten, also der Unterschenkel- und Plantarbeuger und der Hüftmuskeln. Die Kleine bewegt seit den ersten galvanischen Sitzungen ihre Beine entschieden lebhafter und mehr, sowohl auf periphere Reize, als spontan, während die Reflexerregbarkeit, die bei der ersten Untersuchung einige Steigerungsphänomene zeigte, eher abgenommen hat. Eine faradische Untersuchung hat bisher noch nicht wieder stattgefunden.

Die Anamnese, sowie die Symptome lassen in diesem Falle wohl keinen $\mathrm{Z}_{w e i f e l}$ darüber, dass die Ursache der Lähmung eine centrale, eine unter der Geburt entstandene Läsion des Rïckenmarkes - wahrscheinlich in dem Abschnitte der Lendenanschwellung - ist.

In der mir zugänglichen Literatur habe ich über diesen Gegenstand nur wenig Aufklärung gefunden. Die meisten Autoren beschränken sich auf eine Besprechung der unter der Geburt durch Druck auf die peripheren Nervenstämme entstandenen Lähmungen, meist also der oberen Extremitäten. So behandelt Duchenne 1), der den "Paralysies obstétricales" einen eigenen Abschnitt gewidmet hat, ausser den Facialis - Lähmungen, nur die Lähmungen der oberen Extremitäten, bewirkt durch Druck auf den Plexus brachialis, entweder durch die Zange (!), oder durch die Hand des Geburtshelfers bei schwieriger Armlösung, oder

1) De l'électrisation localisée. 3e édition. Paris 1872. Deuxième partie. Chap. I. Article 3. Paralysies obstétricales infantiles. 
schwieriger Extraction des Rumpfes nach der Geburt des Kopfes. Ebenso Seligmüller. ${ }^{1}$ ) Hennigㄹ) allein erwähnt eine in wenigen Tagen vorübergehende Parese der unteren Extremitäten bei solchen Kindern, welche mit dem Steisse voran geboren wurden und einen Druck auf den Nervus ischiadicus erlitten.

An Beispielen durch Läsion der Nervencentren unter der Geburt entstandener Lähmungen, beziehentlich solcher Verletzungen der Wirbelsäule und des Rückenmarkes, die bei Erhaltung des Lebens eine Lähmung erzeugt haben würden, sind mir nur folgende aufgestossen.

Ma uth $n \in r^{3}$ ) theilt einen Fall von einem neugeborenen wohlgebildeten Kinde mit, welches wegen ungünstiger Lage bei der Geburt an den Füssen mühsam herausbefördert worden war und bald nach der Geburt an den unteren Extremitäten gelähmt wurde. Er sucht die Ursache dieser Lähmung in einer Spinalapoplexie, ,wie sie oft die Folge einer durch gewaltsame Entbindung geschehenen Zexrung des Rückenmarkes sei“". Doch wurde der Fall nur ambulatorisch behandelt, und der weitere Verlauf nicht beobachtet.

In einem zweiten Falle, den Mauthner ebenfalls hierher rechnet, wurde ein neugeborenes Kind plötzlich ohne bekannte Ursache an beiden Händen gelähmt, wobei der Kopf rückwärts gezogen wurde, und die Nackenwirbel bei Druck empfindlich waren. Auf Blutegel ins Genick, warme Einwickelung und Einreibung mit reizenden Salben trat wohl bald einige Beweglichkeit ein. Später aber kamen Fraisen hinzu. Der Ausgang blieb auch hier unbekannt.

Parrot ${ }^{4}$ ) berichtet folgenden Fall. Bei einer Erstgebärenden hatte eine Hebamme das mit den Füssen vorliegende Kind durch einen starken Zug an den Beinen extrahirt und dabei ein Krachen gehört, das von den oberen Theilen des Kindeskörpers zu kommen schien. Das Kind kam scheintodt zur Welt und wurde erst nach einer Viertelstunde wieder belebt. Die Lähmung wurde nicht

1) Berliner klinische Wochenschrift 1874, Nr. 40.

2) Krankheiten des Kindes, S. 320. Leipzig 1855.

3) Krankheiten des Gehịns und Rückenmarkes bei Kindern, S. 403. Wien 184.

4) Union médicale 1870, Janvier 27, citirt: Leyden, Klinik der Rückenmarkskrankheiten, Bd. II, S. 88 , und Nadaud (s. a.). 
sogleich bemerkt. Die Arme hingen schlaff herunter, die Beine waren unbeweglich, die Sensibilität erschien erhalten (?), Reflexbewegungen erfolgten auf Reizung der Haut. Harn- und Stuhlverhaltung. Tod am 6. Tage. Autopsie: in der Höhe des 6. und 7. Cervicalnerven eine Zerreissung der Rückenmarkshäute und vollständige Zerreissung des Rückenmarkes selbst; zwischen beiden Enden desselben lag ein Blutgerinnsel, welches den ganzen Kanal der Breite nach ausfüllte. Die Wirbelsäule unverletzt (?).

Nadaud ${ }^{1}$ ) giebt die Geschichte einer sehr schwierigen, durch die sich ablösende Thätigkeit dreier Aerzte mittels der Zange beendigten Geburt. Das grosse Kind war scheintodt, wurde aber bald wieder belebt. Ausser einer Lähmung des Nervus facialis rechterseits bestand eine Lähmung beider oberen sowohl, als unteren Extremitäten. Wie lange das Kind lebte, wird nicht gesagt. Die Section wurde nicht gestattet. Doch ist an dem Vorhandensein einer centralen Läsion wohl nicht zu zweifeln. Nadaud erwähnt ausserdem noch die vorstehende Beobachtung von Parrot, sowie einen von Guéniot in derselben Nummer der Union mitgetheilten Fall von Zerreissung des dritten Halswirbelkörpers mit Ruptur des Rückenmarkes. Der Kopf war bei der Geburt abgewichen, und die sehr energischen Tractionen hatten fast ausschliesslich den Hals des Kindes getroffen.

Ahlfeld ${ }^{2}$ zeigte in der Gesellschaft für Geburtshülfe in Leipzig ein Stück einer Brustwirbelsäule, an welcher bei der Extraction der Frucht an den Füssen bei dem Versuche, den Kopf herauszuheben, zwei Wirbelkörper getrennt worden waren. Das Kind hatte neun Tage gelebt, und vor dem Tode war von der Läsion nichts bemerkt worden.

Ruge ${ }^{3}$ ) beobachtete unter 64 Fällen von Extraction an den Füssen acht Male eine Ruptur der Wirbelsäule, d. h. eine Trennung in der Substanz eines Wirbels an der Epiphysenlinie, meist nur des Wirbelkörpers mit Zerreissung des Ligamentum vertebrale anticum (ein $\mathrm{Mal}$ diese isolirt, wahrscheinlich als Anfang der Wirbelruptur, zwei Male Doppelruptur). Im Grunde der Ruptur-

1) Paralysies obstétricales des nouveau-nés. Paris 1872.

2) Dieses Archiv 1873, Bd. V, S. 161.

3) Ueber Verletzungen des Kindes durch die Extraction bei ursprünglicher oder durch die Wendung herbeigeführter Beckenendlage. Zeitschrift für Geburtshülfe und Frauenkrankheiten 1876, Bd. I, S. 68 ff. 
stelle erschien fast regelmässig das mit Blutextravasat bedeckte Rückenmark. Obwohl es nicht ausdrücklich gesagt ist, muss ich annehmen, dass sämmtliche Kinder todt oder sterbend zur Welt kamen. Bezüglich des Grades der Geburtserschwerung befanden sich unter den acht Fällen fünf normale, ein unbekanntes, zwei verengte Becken; zwei sehr grosse und sechs normale, beziehentlich unternormale Kinder. Die Verletzungen betrafen immer die Halsoder oberen Brustwirbel, waren also wahrscheinlich bei der Extraction des Kopfes mit Erhebung des Rumpfes entstanden. Ausser diesen Rupturen der Wirbelsäule erwähnt Ruge noch einen Fall von schwieriger Extraction nach der Wendung wegen Ruptur des Uterus, bei engem Becken und grossem Kinde, in welchem durch die Verschiebung der Squama ossis occipitis nach unten und vorn der Längsdurchmesser des Foramen magnum um $0,5 \mathrm{~cm}$ verkürzt war. In der an diesen Vortrag in der gynäkologischen Gesellschaft sich anschliessenden Discussion kamen noch zwei Fälle von Wirbelsäulenruptur, resp. Epiphysentrennung an der Squama occipitis bei der Extraction an den Füssen zur Sprache. Und in einer Anmerkung berichtet Ruge uiber ein wenige Tage vorher secirtes Mädchen, bei dem sich als Folge des nach der Wendung bei engem Becken, vor der Expression des Kopfes, vergeblich am Rumpfe ausgeübten Zuges folgende Verletzungen fanden: Wirbelsäulenzerreissung; Zerreissung der Medulla spinalis, fast vollständig; grosser Bluterguss im Cavum abdominis.

In meinem Falle ist eine Ruptur der Wirbelsåule, beziehentlich des Ruickenmarkes wohl mit Sicherheit auszuschliessen. Ein Mal war, wie schon gesagt, die Extraction ohne übermässigen Kraftaufwand bewirkt, der Zug hatte nur in der Richtung der Wirbelsäulenaxe stattgefunden. Dann war, trotz sorgfältiger wiederholter. Untersuchung, an der Wixbelsäule nirgends eine krankhafte Veränderung zu entdecken. Endlich wiurden bei einer solchen Verletzung, im Falle uiberhaupt das Leben erhalten wäre, schwerere Folgen, wie intensivere Blasenlähmung, Decubitus u. s. w. nicht ausgeblieben sein. Mir scheint es unzweifelhaft, dass die Lähmung durch einen während der Geburt entstandenen Bluterguss in den Wirbelkanal bedingt wurde, entweder zwischen diesem und der Dura mater, oder vielleicht wahrscheinlicher im sogenannten Arachnoidealsacke, resp. im Subarachnoidealraume. Die anfängliche Ausbreitung der Lähmungserscheinungen liesse sich durch eine Fortdauer der Blutung nach der Geburt, resp. 
eine Nachblutung ungezwungen erklären. Die Ursache der Blutung suche ich nur zum Theil in der, bei keiner Extraction an den Füssen wohl ganz zu vermeidenden, Zerrung oder Quetschung der Wirbelsäule, sondern vorzugsweise in der, durch die mehrfache Umschlingung der dünnen Nabelschnur veranlassten Circulationsstörung, deren Intensität sich durch die jähe Abschwächung des Fötalpulses kund gab. Die langsame, wenigstens theilweise Besserung des Zustandes wäre als ein Zeichen der allmäligen Resorption des Blutextravasates anzusehen, während der Fortbestand der gänzlichen Lähmung in den beiderseitigen Cruralisund zum Theil auch den Peroneus-Gebieten vielleicht auf eine beschränkte (secundäre) Affection der grauen Vordersäulen des Rückenmarkes bezogen werden müsste. Ich glaube diese Ansicht von dem Ursprunge der Lähmung, sowohl durch anatomische, als durch klinische Thatsachen stützen zu können.

Ueber die Häufigkeit spinaler Blutungen unter der Geburt ohne mechanische Insultation der Wirbelsäule, die ja bei der Mehrzahl der Schädelgeburten ausgeschlossen werden kann, hat mich die eigene Erfahrung genügend belehrt. Unter 161 in den Jahren 1849 bis 1861 in meiner Klinik, grösstentheils durch meinen verstorbenen Collegen $W$ eber ausgeführten Sectionen wurde 81 Male der Wirbelkanal geöffnet, und wurden in diesen 81 Fällen 33 Male, und zwar 19 Male sehr ausgedehnte und massenhafte Blutextravasate in demselben gefunden, 23 Male allein auf der Aussenfläche der Dura mater, bisweilen die Ursprünge der abgehenden Nerven umfilzend, 4 Male zugleich im sogenannten Arachnoidealsacke, $1 \mathrm{Mal}$ zugleich im Subarachnoidealraume, $4 \mathrm{Mal}$ allein im Arachnoidealsacke, 1 Mal allein im Subarachnoidealraume. Eine Verletzung des Wirbelkanales wurde niemals wahrgenommen. Neben den Blutergüssen im Arachnoidealsacke oder Subarachnoidealraume des Rückenmarkes fanden sich immer auch intracranielle, meist umfangreiche meningeale Blutextravasate, während sie bei Blutergüssen zwischen Dura mater und Wirbelkanal in reichlich einem Drittheile der Fälle fehlten. Unter den 33 Kindern befanden sich 23 rechtzeitige und 10 frühzeitige, meist bei engem Becken, nach spontaner oder künstlich eingeleiteter Frühgeburt. ${ }^{1}$ )

1) Auf die grössere Zerreisslichkeit der Gefässwandungen bei Frühgeborenen, in Folge deren alle Circnlationsstörungen, mögen sie durch ört- 
Von diesen 33 Kindern kamen 13, und zwar 8 rechtzeitige und 5 frïhzeitige, sterbend oder todt zur Welt. Sechs derselben waren in Schädellage geboren, 4 natürlich, 2 mittels der Zange entwickelt; darunter 5 mit Blutergüssen im Arachnoidealsacke des Rückenmarkes, die in vier Fällen eine bedeutende Mächtigkeit erreicht hatten, einmal zugleich im Subarachnoidealraume. Sieben Kinder waren an den Füssen extrahirt, vier bei ursprünglicher Fusslage, drei nach der Wendung auf die Füsse. Bei dreien derselben fanden sich starke Blutextravasate im Arachnoidealsacke des Rückenmarkes, bei ein em eine beschränkte subarachnoideale Blutung.

Unter den 20 lebend geborenen Kindern befanden sich 15 rechtzeitige und 5 frühzeitige. Sie waren sämmtlich in Schädellage geboren, 16 natürlich, 4 mit der Zange extrahirt. Fünf darunter kamen mehr oder weniger scheintodt zur Welt; von acht ist ausdrücklich das Gegentheil vermerkt.

Fünf unter diesen Kindern, darunter vier frühzeitige, starben schon in den ersten 24 Stunden. Das rechtzeitige Kind war von der Mutter in einem Anfalle von Urämie geboren, schrie zwar sogleich, blieb aber auffällig cyanotisch und starls nach 12 Stunden. Bei der Section fanden sich, neben einem mässigen Blutergusse zwischen Wirbelkanal und Dura mater, starke intracranielle Blutergrüsse. Von den vier frühzeitigen Kindern starben zwei unter den Erscheinungen gestörter Respiration mit raschem Collaps, eines an Trismus, eines ohne nähere Angabe der vorausgegangenen Symptome. Bei allen wies die Section umfangreiche Blutextravasate zwischen Wirbelkanal und Dura mater, ein Mal zugleich im Arachnoidealsacke des Rïckenmarkes nach; daneben bei zweien starke intracranielle Blutergüsse, während diese bei den übrigen zweien ein $\mathrm{Mal}$ sehr gering waren, das andere Mal ganz fehlten. Bei dem an Trismus gestorbenen Kinde, welches nach einer natürlichen Geburt nicht scheintodt gewesen war, fand sich, ausser einem geringen intracraniellen Blutergusse und den Blutextravasaten zwischen Wirbelkanal und Dura mater, welche vorzugsweise die Cervical- und Lumbalgegend einnahmen, das Rücken-

liche Quetschung, oder Hindernisse im Placentarkreislaufe bedingt sein, mit grösserer Gefahr für das Kind verknüpft sind, habe ich schon früher in meiner Arbeit über den Werth der künstlich eingeleiteten Frühgeburt (dieses Archiv, Bd. II, S. 206 ff.) aufmerksam gemacht. 
mark im Centrum in beiden Hälften breiig erweicht, von röthlichgrauer Farbe, am stärksten im Cervicaltheile.

Zwölf Kinder starben zwischen dem zweiten und zehnten Lebenstage, darunter eilf nach voraufgegangenen klonischen oder tonischen Krämpfen, bisweilen ausgesprochenem Trismus, eines an Pyämie in Folge einer Vereiterung der durch die Zangenlöffel auf der Kopfhaut erzeugten Druckstellen. Bei allen zwölf fanden sich mehr oder weniger rostfarbig verfärbte Blutextravasate zwischen dem Wirbelkanale und der Dura mater, fünf Male von sehr bedeutender, drei Male von mässiger, vier Male von geringer Ausdehnung; daneben in sieben Fällen intracranielle Blutergüsse, vier Male von beträchtlichem, zwei Male von mässigem, ein Mal von geringem Umfange; in den übrigen fünf Fällen fehlten sie. Bei einem der an Trismus gestorbenen Kinder, einem natürlich und lebensfrisch geborenen Knaben, welcher am achten Tage erkrankte und nach 24 Stunden starb, fand sich ausser einem beträchtlichen schmierigen, rostfarbigen Blutextravasate in der Schädelhöhle, einem ebenfalls beträchtlichen, halb-weich geronnenen, rostfarbig verfärbten Blutextravasate nebst schmierigem flüssigen Blute auf der Aussenfläche der Dura mater in der ganzen Länge des Wirbelkanales, die Marksubstanz des Rückenmarkes, besonders in der Cervicalgegend, sehr matsch, von schmutzig gelber Farbe, obwohl die mikroskopische Untersuchung eine pathologische Veränderung nicht erkennen liess.

Drei Kinder starben nach dem 14. Lebenstage. Ein frühzeitig geborenes Kind starb 15 Tage alt. Es war nach der Geburt kaum scheintodt gewesen. Aber schon nach einigen Tagen fiel die eigenthümliche Haltung der Beine auf. Beide Unterschenkel und Füsse waren dauernd stark flectirt, und beim Versuche, sie zu strecken, fühlte man eine beträchtliche Spannung der Beugemuskelsehnen, und das Kind wurde sehr unruhig (Warme Bäder; kalte Umschläge auf den Kopf; Calomel.) In den letzten Lebenstagen wurde es wiederholt von Convulsionen befallen. Die Section ergab, neben kleinen Blutergüssen in der Schädelhöhle und einer geringen Ansammlung klaren Serums in den etwas erweiterten Hirnventrikeln, ebenfalls nur beschränkte Blutextravasate zwischen den Rïckenwirbeln und der Dura mater, aber eine centrale Erweichung des Rückenmarkes, besonders in seinem oberen Theile. - Bei einem zweiten, rechtzeitig geborenen Kinde, welches am 16. Lebenstage ohne voraufgegangene 
Zuckungen starb und bis zum Abend vorher sich anscheinend wohl befunden hatte, fand man bei der Section ebenfalls nur beschränkte rostfarbige Blutextravasate zwischen Wirbelkanal und Dura mater, welche die Ursprünge der abgehenden Nerven umfilzten, ohne intracranielle Blutergüsse. - Ein drittes, gleichfalls rechtzeitig geborenes Kind starb erst am 28. Lebenstage. Es war mit der Zange extrahirt, nicht scheintodt, nur mit einer leichten Facialis-Lähmung behaftet, die sich bald verlor. Am 18. Tage bemerkte man eine Anschwellung und Lähmung des rechten Armes. Dem Tode gingen Spuren von Trismus vorher. Die Section ergab, neben geringen, rostfarbig verfärbten Blutextravasaten in der Schädelhöhle, einen sehr umfangreichen Bluterguss zwischen Wirbelkanal und Dura mater.

Eine Extravasation von Blut in die Substanz des Riuckenmarkes selbst haben wir niemals gefunden. Auch in seinen früheren. Beobachtungen scheint Weber ${ }^{1}$ ) sie nicht gesehen zu haben. Sie muss bei Neugeborenen hier jedenfalls noch viel seltener vorkommen, als im Gehirn, in dem wir, neben den gewöhnlichen Meningenalblutungen, einige wenige Male Capillarapoplexien und kleineren apoplectischen Herden begegnet sind. Ueberhaupt sind die erwähnten drei Fälle von Erweichung des Rückenmarkes die einzigen, in denen eine gröbere Texturabweichung - ob primäre, oder secundäre, muss ich dahingestellt sein lassen - an demselben sichtbar war. Die sonst wahrgenommenen Veränderungen beschränkten sich auf einfache Hyperämie, oder Anämie und geringe Unterschiede in der Consistenz.

Die vorstehenden anatomischen Thatsachen beweisen zunächst allerdings nur, dass Blutungen der Rückenmarkshäute unter der Geburt keine seltenen Erscheinungen sind, und dass es selbst zur Erzeugung massenhafter Extravasate eines besonderen, die Wirbelsäule treffenden Trauma nicht bedarf. Dass diese Blutergüsse im Stande seien, direct das Leben zu vernichten, möchte ich bezweifeln. Wo die damit behafteten Kinder todt oder sterbend zur Welt kamen, ist die unmittelbare Ursache des Todes wahrscheinlicher in anderen gleichzeitigen Störungen zu suchen. Ueber die Folgen solcher Blutungen aber, wenn das Leben die Geburt überdauerte, geben die von mir mitgetheilten Fälle nur unvollkom-

1) Beiträge zur pathologischen Anatomie der Neugeborenen. (Erste Lieferung: Kopf and Rücken. Kiel 1851.) 
menen Aufschluss. Mit Ausnahme des einen Falles, in welchem die einige Tage nach der Geburt bemerkte Contractur der Unterschenkel und Füsse darauf bezogen werden kann, sind niemals Lähmungserscheinungen an den unteren Extremitäten erwähnt. Indess glaube ich, dass diese, zumal wenn das Leben nur von kurzer Dauer ist, leicht übersehen werden können. War ja doch in dem Falle von Parrot, in welchem das Rückenmark zerrissen war, die Lähmung nicht gleich bemerkt, und in dem Falle von Ahlfeld, in welchem die Section eine Trennung zweier Brustwirbelkörper nachwies, während der neun Tage, welche das Kind lebte, die Verletzung unerkannt geblieben. Immer werden abgesehen von primären Läsionen des Rückenmarkes selbst - nur solche Blutextravasate im Wirbelkanale, welche durch ihre Mächtigkeit oder durch ihren Sitz einen beträchtlichen Druck auf dasselbe auszuüben im Stande sind, eine vollständige Paraplegie bewirken. Doch würde diese gewiss häufiger beobachtet werden können, wenn nicht unter den Bedingungen, welche während der Geburt zu ihrer Entstehung Anlass geben, in der Regel das Leben des Kindes verloren ginge.

Dass indess bei Kindern während der ersten Lebenszeit eine Lähmung der unteren Extremitäten in Wirklichkeit nicht ganz so selten vorkommt, als man gewöhnlich annimmt, scheint mir aus Billard's ') Erfahrungen hervorzugehen. Er spricht zunächst von einer dem Gehirne bei neugeborenen Kindern eigenthümlichen Veränderung, einer Art von allgemeiner oder örtlicher Erweichung, die, weit entfernt, die Charaktere einer Entzündung zu besitzen, vielmehr die Zeichen der Auflösung, fast könnte man sagen, der Fäulniss zeige. Er habe diese Erweichung des Gehirns oft bei neugeborenen Kindern gefunden, welche fast gleich nach der Geburt gestorben waren, was ihn glauben lasse, dass sie während des Aufenthaltes des Kindes im Mutterleibe stattgefunden habe. - Daneben erwähnt er eine allgemeine und örtliche Erweichung des Rückenmarkes bei Neugeborenen, die entweder ungefärbt, oder mit einer Blutergiessung vermischt sei. Die allgemeine Erweichung des Rückenmarkes sei selten ohne Erwei-

1) Krankheiten der Neugeborenen und Säuglinge, nach neuen klinischen und pathologisch-anatomischen, in dem Hospital der Findelkinder angestellten Beobachtungen geschildert. Aus dem Französischen. Neue Auflage, bereichert durch Nachträge nach der zweiten, von Ollivier (d'Angers) besorgten Auflage des Originales. Weimar 1829 und 1837. 
chung des Gehirnes vorhanden; häufiger das Gegentheil. Diese Erweichung des Rückenmarkes zeige dieselben Charaktere, wie die des Gehirns. In den Fällen, wo man diese Veränderungen treffe, habe das Kind gewöhnlich nur einige Tage gelebt, sein Schreien sei erstickt gewesen, es habe sich fast gar nicht bewegen können, seine Glieder seien welk, seine Integumente violett, sein Gesicht keiner Bewegung fähig. Die Desorganisation des Gehirns allein gebe gewöhnlich zu weit geringeren functionellen Störungen. Anlass, als wenn die Hirn-Rückenmarksaxe krank sei. Immer scheine die Erweichung sich vom Gehirne aufs Rückenmark auszubreiten, und die Symptome seien um so stärker, je weiter die Desorganisation des Rückenmarkes vorgeschritten sei. Er habe oft bei neugeborenen Kindern das Gehirn ganz zerstört gefunden, ohne dass ein äusseres Zeichen während des Lebens diese Zerstörung habe vermuthen lassen. Dagegen sei es niemals bei der Erweichung des Rïckenmarkes so gewesen. - Die partielle Erweichung des Rïickenmarkes komme ziemlich oft vor, am häufigsten in dem oberen Drittheile, nach dem unteren Ende zu abnehmend. Auch eine partielle Verhärtung werde beobachtet. So sei die Lendenauftreibung oft sehr hart, während das Uebrige des Rïckenmarkes leicht zu einer breiartigen Masse gemacht werde. Besondere Symptome, welche dieser Veränderung des Markes entsprochen hätten, habe er indess nicht gesehen. Bisweilen finde man auch eine Erweichung des unteren Drittheiles, während der obere Theil von einer festen Consistenz sei. Bei einem Kinde, welches im Alter von sechs Monaten eine Meningitis gehabt habe und im 18. Monate gestorben sei, habe er eine sehr beträchtliche Erweichung der unteren Auftreibung des Rückenmarkes gefunden. Das Kind war an den unteren Gliedmaassen gelähmt, welche es gar nicht unterstützen konnten, und die es nachschleppte, wie rachitische Kinder thun. Er habe oft diese Lähmung der unteren Gliedmaassen bei Kindern bemerkt, welche bereits das Alter erreicht hätten, wo sie gewöhnlich gehen könnten. Es rührt dieser Fehler, seiner Meinung nach, von einer Affection des Rïckenmarkes, vielleicht einer chronischen Hydro-Rhachis her. Uebrigens werde diese Meinung durch zahlreichere Untersuchungen bestätigt werden mïssen, als diejonigen, welche er vorzunehmen im. Stande gewesen sei.

Einen weiteren Einblick, vom klinischen Standpunkte aus, in diese noch dunklen Verhältnisse eröffnet eine Arbeit des aus- 
gezeichneten englischen Orthopäden W. J. Little. ${ }^{1}$ ) Er fand unter 47 Fällen von ,Spastic rigidity“", d. h. paralytischer, resp. neuropathischer Contraction mehr oder weniger ausgedehnter Muskelgruppen, 22 Male ausschliesslich beide untere Extremitäten, 16 Male daneben eine oder beide obere Extremitäten, und neun Male untere und obere Extremität nur ei ner Seite afficirt. Von den Kranken standen beim Eintritte in die Behandlung $20 \mathrm{im}$ Alter von ein bis fünf Jahren, 18 im Alter von sechs bis zehn Jahren, 9 waren über zehn Jahre alt. Indem Little durch sorgfältige Erkundigungen bei den Eltern oder den sonstigen Angehörigen der Kranken die Entstehung des Leidens festzustellen sich bemühte, gelangte er zu der Ueberzeugung, dass die Affection durch eine unter der Geburt entstandene Läsion der Centralorgane des Nervensystems bedingt sei, vorzugsweise des Rïckenmarkes, dessen Läsionen, vermöge seiner vollendeten Entwickelung zu dieser Lebenszeit, niemals ohne Symptome verliefen, im Gegensatze zu den Läsionen des unentwickelteren Gehirns, wie schon von Ollivier ${ }^{2}$ ) mit Recht hervorgehoben sei. Doch befanden sich unter Little's Kranken einige, bei welchen, neben den Rückenmarkssymptomen, früher oder später deutliche Störungen der Functionen von Gehirnnerven, wie erschwertes Sprechen, Schlingbeschwerden, sowie Störungen der Intelligenz bemerkt wurden. Auch waren in reichlich einem Sechstel der Fälle, wie schon erwähnt, die Krankheitserscheinungen auf eine Körperseite beschränkt. Als die Quelle der Läsion betrachtet Lit tle, unter Berufung auf die von W eber, Hecker u. A. veröffentlichten Sectionsresultate, cerebrospinale Blutergüsse, bedingt durch Störung, beziehentlich Unterbrechung des Placentarverkehres, seltener durch directe mechanische Insultation. Er verweist in dieser Beziehung auf das überwiegend häufige Befallensein der unteren Extremitäten, die doch von dem unteren Abschnitte des Rückenmarkes, also demjenigen Theile des Cerebrospinalsystems innervirt würden, der im

1) On the influence of abnormal parturition, difficult labours, premature birth and asphyxia neonatorum on the mental and physical condition of the child, especially in relation to deformities. Communicated by Dr. Tyler Smith. Transactions of the obstetrical society of London, 1862, Vol. III, p. 293--344.

2) Traité de la moelle épinière et de ses maladies. 2e édition. A Paris 1827. Vol. I, p. 127-128. (Little hat wahrscheinlich nach der dritten, 1837 erschienenen Ausgabe citirt.) Ollivier beruft sich an dieser Stelle ausdrücklich auf Billard's Erfahrungen. Vergl. Billard, l. c. 
106 Litzmann, Ein Beitrag zur Kenntniss der spinalen Lähmung u. s. w.

Vergleiche mit den übrigen am seltensten einer mechanischen Schädigung ausgesetzt sei. Aus den beigefügten Tabellen ergiebt sich, dass ron den 38 Kranken, bei welchen die unteren Extremitäten entweder ausschliesslich, oder neben einer oder beiden oberen Extremitäten afficirt waren, nur 14 rechtzeitig, die übrigen 24 dagegen vor der Zeit das Licht der Welt erblickt hatten. Nur eines der Kinder war an den Füssen (nach der Wendung) extrahirt, alle übrigen scheinen in Kopflage - darunter eines in Gesichtslage - geboren zu sein. Bei acht derselben sollte die Geburt, wenn auch natürlich, doch schwer und langsam verlaufen sein; drei Kinder waren mittels der Zange extrahirt. In etwa der Hälfte der Fälle waren die Kinder mehr oder weniger scheintodt zur Welt gekommen - nur in drei Fällen wird der Scheintod ausdrücklich geläugnet - und in fast einem Drittheile der Fälle wird eine grosse Schwäche während der ersten Lebenszeit betont. Von zehn Kranken liess sich feststellen, dass sie in den ersten Lebenstagen an Krämpfen gelitten hatten; bei acht waren schon in frühester Lebenszeit Bewegungsstörungen bemerkt, wie Steifheit, gekreuzte Haltung der Beine, Unvermögen, sie von einander zu entfernen, Unfähigkeit zu sitzen, zu stehen, Neigung im Liegen auf die Seite zu rollen; bei zweien hatte sich schon früh Neigung zu Harnverhaltung gezeigt.

Es scheint - wenigstens so weit ich den Gegenstand in der Literatur habe verfolgen können -, dass die Arbeit von Little keine weiteren Forschungen in der von ihm angedeuteten Richtung angeregt hat. Wenn ich jetzt meine Beobachtungen, trotz ihrer unfertigen Gestalt, veröffentliche, so geschieht es in der Hoffnung, dass diejenigen, welche im Stande sind, die Lücken derselben zu. ergänzen und zur Aufhellung der dunkel gebliebenen Punkte beizutragen, dadurch veranlasst werden möchten, ihre Erfahrungen mitzutheilen. 